\title{
On the Relative Distances of Seven Points in a Plane Convex Body
}

\author{
Antal Joós* and Zsolt Lángi**
}

November 24, 2006

* Partially supported by the Hung. Nat. Sci. Found. (OTKA), grant no. T043556. ** Partially supported by the Hung. Nat. Sci. Found. (OTKA), grant no. T043556 and T037752 and by the Alberta Ingenuity Fund.

\begin{abstract}
Let $C$ be a convex body in the Euclidean plane. The relative distance of points $p$ and $q$ is twice the Euclidean distance of $p$ and $q$ divided by the Euclidean length of a longest chord in $C$ with the direction, say, from $p$ to $q$. We prove that, among any seven points of a plane convex body, there are two points at relative distance at most one, and one cannot be replaced by a smaller value. We apply our result to determine the diameter of point sets in normed planes.
\end{abstract}

Mathematics Subject Classification (2000): 52A40, 52A38, 52A10

Key words: convex body, normed space, relative distance, packing

\section{Introduction}

The focus of the paper is systems of "far" points in normed spaces in general, and normed planes in particular. Given $k \geq 3$, we look for sets of $k$ points in a convex body $C$ with minimum pairwise distance as large as possible. This is equivalent to packing $C$ by congruent homothetic copies of $C$.

Let $p$ and $q$ be points in $n$-dimensional Euclidean space $\mathbb{E}^{n}$. Let $[p, q],(p, q),|p q|$ and $\overrightarrow{p q}$ denote, respectively, the closed and the open segment, the distance and the vector with initial point $p$ and terminal point $q$. Furthermore, if $P$ is a simple polygon with edges $\left[a_{1}, a_{2}\right],\left[a_{2}, a_{3}\right], \ldots,\left[a_{n}, a_{1}\right]$, we use the notations $P=\left[a_{1}, a_{2}, \ldots, a_{n}\right]$ and int $P=\left(a_{1}, a_{2}, \ldots, a_{n}\right)$. We use the usual notations card $A, \operatorname{conv} A, \operatorname{int} A, \operatorname{bd} A$ for the cardinality, the convex hull, the interior and the boundary of a set $A$, respectively. We denote the family of plane convex bodies by $\mathcal{C}$ and the family of centrally 
symmetric plane convex bodies by $\mathcal{M}$. For simplicity, we call a plane convex body an oval, and identify a point and its position vector.

Let $C \subset \mathbb{E}^{n}$ be a convex body, and denote by $r$ and $s$ points in $C$ such that $\overrightarrow{r s} \| \overrightarrow{p q}$ and $|r s| \geq\left|r^{\prime} s^{\prime}\right|$ where $\left\{r^{\prime}, s^{\prime}\right\} \subset C$ and $\overrightarrow{r^{\prime} s^{\prime}} \| \overrightarrow{p q}$. The $C$-length of $[p, q]$, or equivalently, the $C$-distance of $p$ and $q$ is $2|p q| /|r s|$, and we denote it by $d_{C}(p, q)$ (see also [11]). If the convex body $C$ is obvious, we may use the terms relative distance of $p$ and $q$ or relative length of $[p, q]$. Observe that for any convex bodies $D \subset C$ and points $p, q$ we have $d_{C}(p, q) \leq d_{D}(p, q)$. It is a well-known fact that the unit ball of the normed space with norm $d_{C}(0, x)$ is $\frac{1}{2}(C-C)$.

Let $C \in \mathcal{C}$ and $k \geq 2$. Then compactness arguments yield that there is a greatest value $f_{k}(C)$ such that $C$ contains $k$ points in pairwise $C$-distances at least $f_{k}(C)$. Let $f_{k}=\min _{C \in \mathcal{C}}\left\{f_{k}(C)\right\}$ and $F_{k}=\max _{C \in \mathcal{C}}\left\{f_{k}(C)\right\}$. By Blaschke's Selection Theorem these values exist.

Numerous results appeared about the values $f_{k}(C), f_{k}$ and $F_{k}$. Here we list a few. Doliwka and Lassak [4] proved that, among any five boundary points of an oval, there is two at relative distance at most $\sqrt{5}-1 \approx 1.236$ and that the value $\sqrt{5}-1$ cannot be replaced by a smaller one. Böröczky and Lángi [2] showed that the result of Doliwka and Lassak remains true if we consider arbitrary points of the oval. In other words, $F_{5}=\sqrt{5}-1$. They also proved that $F_{6}=2-\frac{2 \sqrt{5}}{5} \approx 1.106$, and conjectured that $F_{7}=1$. We verify their conjecture.

Theorem 1 Let $C \in \mathcal{C}$ and let $a_{1}, \ldots, a_{7}$ be points in $C$. Then $d_{C}\left(a_{i}, a_{j}\right) \leq 1$ for some $i \neq j$.

Let us call an oval $C$ optimal if it contains seven points at the minimum pairwise relative distance equal to one. In this case we say that the points fit $C$. The problem arises naturally to determine the optimal ovals and the set of points fitting them. We present the following three examples.

A result of Gołąb [6] states that there is an affine regular hexagon $H$ inscribed in $C$ for every $C \in \mathcal{M}$. The vertices and the centre of $H$ fit $C$, and hence, $C$ is optimal. Another example: any parallelogram $P$ contains many sets of seven points at pairwise $P$-distances at least 1 . Any oval $C \subset P$ containing such a set is optimal.

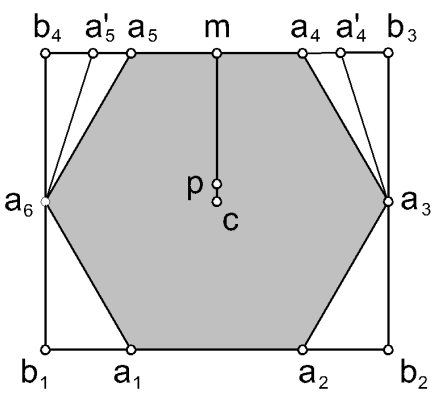

Figure 1

The third example is the following. Let $H=$ $\left[a_{1}, a_{2}, \ldots, a_{6}\right]$ be a regular hexagon and $S=\left[b_{1}, b_{2}, b_{3}, b_{4}\right]$ be a rectangle circumscribed about $H$ such that $\left[a_{1}, a_{2}\right] \subset\left[b_{1}, b_{2}\right]$ and $a_{1} \in\left[b_{1}, a_{2}\right]$. Let $c$ be the centre of $H$ and $m=\left(b_{3}+b_{4}\right) / 2$. Let $a_{4}^{\prime} \in\left(b_{3}, a_{4}\right)$ and $a_{5}^{\prime} \in\left(a_{5}, b_{4}\right)$ such that $\left|a_{4} a_{4}^{\prime}\right|=\left|a_{5} a_{5}^{\prime}\right|$ 
and let $p \in(c, m)$; cf. Figure 1. Finally, let $C=\left[a_{1}, a_{2}, a_{3}, a_{4}^{\prime}, a_{5}^{\prime}, a_{6}\right]$. If $p$ is close enough to $c$, all the pairwise $C$-distances of the vertices of $C$ and $p$ are at least one. We collect our results about optimal ovals and fitting sets of points in Theorem 2.

Theorem 2 Let $C \in \mathcal{C}$ such that $Q=\operatorname{conv}\left\{a_{1}, a_{2}, \ldots, a_{7}\right\} \subset C$ and $d_{C}\left(a_{i}, a_{j}\right) \geq 1$ for all $i \neq j$.

2.1 If $C$ is strictly convex then $Q$ is an affine regular hexagon with some $a_{i}$ as centre.

2.2 If $\operatorname{card}\left(\mathrm{bd} Q \cap\left\{a_{1}, a_{2}, \ldots, a_{7}\right\}\right) \neq 6$ then there is a parallelogram $P$ such that $C \subset P$ and $d_{P}\left(a_{i}, a_{j}\right) \geq 1$ for all $i \neq j$.

Using the idea of [5] (see also [11] and Theorem of [10]), we reformulate our theorems.

Corollary 3 No oval is packed by seven homothetic copies of ratio greater than $1 / 3$.

Corollary 4 Let $C \in \mathcal{C}$ be packed by seven homothetic copies of ratio $1 / 3$ with points $a_{1}, a_{2}, \ldots, a_{7}$ as centres. Let $Q=\operatorname{conv}\left\{a_{1}, a_{2}, \ldots, a_{7}\right\}$.

4.1 If $C$ is strictly convex then $Q$ is an affine regular hexagon with some $a_{i}$ as centre.

4.2 If card ( $\left.\mathrm{bd} Q \cap\left\{a_{1}, a_{2}, \ldots, a_{7}\right\}\right) \neq 6$ then there is a parallelogram $P$ containing $C$ such that $P$ is packed by seven homothetic copies of ratio $1 / 3$ with $a_{1}, a_{2}, \ldots, a_{7}$ as centres.

The following lemma is applied in the proof of Theorems 1 and 2 in Sections 2 and 3 . We present applications of our theorems in Section 4. We note that analogous form of 5.1 has been verified in [9]. Theorem 1 when $Q=\operatorname{conv}\left\{a_{1}, a_{2} \ldots, a_{7}\right\}$ is not a hexagon is a consequence of [9] and Lemma 3 of [2]. In that case we prove Theorem 1 for the sake of Theorem 2 .

Lemma 5 Let $C \in \mathcal{C}, n \geq 6, D=\left[a_{1}, a_{2}, \ldots, a_{n}\right] \subset C$ be a (possibly degenerate) convex $n$-gon and $T \subset D$ be an inscribed triangle of largest area with a side coinciding with a side of $D$.

$5.1 D$ has a side of $C$-length at most one.

5.2 If the $C$-lengths of the sides of $D$ are at least one then $C$ is not strictly convex, and there is a parallelogram $P$ such that $C \subset P$ and the sides of $D$ are of $P$-length at least one.

Proof. Without loss of generality, we assume that $T=\left[a_{1}, a_{2}, a_{i}\right]$ for a suitable $i$. Observe that $D \backslash T$ has a component $W$ with at least three edges. We assume that $\left\{a_{2}, a_{i}\right\} \subset$ bd $W$; that is, $i \geq 5$. As relative distance and area ratio do not change under an affine transformation, we assume that $T$ is an isosceles triangle 
and with right angle at $a_{1}$. Let $b$ be the point such that $S=\left[a_{1}, a_{2}, b, a_{i}\right]$ is a square. Since $T$ is a triangle of maximal area inscribed in $D$, we have $a_{j} \in\left[a_{2}, b, a_{i}\right]$ for $j=3, \ldots, i-1$.

Let $m_{1}=\left(a_{2}+b\right) / 2, m_{2}=\left(b+a_{i}\right) / 2$ and $m=\left(a_{i}+a_{2}\right) / 2$. If $a_{3} \in\left[a_{2}, m_{1}, m\right] \backslash$ $\left[m, m_{1}\right]$ then $d_{C}\left(a_{2}, a_{3}\right) \leq d_{T}\left(a_{2}, a_{3}\right)<1$ and we are done. If $a_{i-1} \in\left[a_{i}, m, m_{2}\right] \backslash$ $\left[m, m_{2}\right]$ then $d_{C}\left(a_{i-1}, a_{i}\right)<1$. We are left with the case $a_{j} \in S_{0}=\left[m, m_{2}, b, m_{3}\right]$ for $3 \leq j \leq i-1$.

In this case $d_{C}\left(a_{j}, a_{j+1}\right) \leq d_{T}\left(a_{j}, a_{j+1}\right) \leq 1$ for $3 \leq j \leq i-1$. This proves 5.1. Moreover, if, for some $3 \leq j \leq i-2$, the points $a_{j}$ and $a_{j+1}$ are not on parallel sides of $S_{0}$ then $d_{C}\left(a_{j}, a_{j+1}\right) \leq d_{T}\left(a_{j}, a_{j+1}\right)<1$. Let us examine the opposite case. Then $i=5$ or $i=6$, and, in the latter case, $a_{3}=m_{1}, a_{4}=b$ and $a_{5}=m_{2}$, which implies that $S \subset C$. If $S \neq C$ then among the points there is two at $C$-distance less than one. If $S=C$ then the pairwise $S$-distances of the points are at least one.

Let us assume that $i=5$ and that, say, $a_{3} \in\left[m_{1}, m\right]$ and $a_{4} \in\left[m_{2}, b\right]$. Let $M$ denote the closed infinite strip containing $S$ and bounded by the line through $a_{1}$ and $a_{2}$ and the line through $b$ and $a_{5}$; cf. Figure 2. From $d_{C}\left(a_{2}, a_{3}\right) \geq 1$, we obtain that $C \subset$ $M$. Let $u, v$ be the endpoints of a maximal chord of $C$ parallel to $\overrightarrow{a_{3} a_{4}}$, and $N$ be the closed strip bounded by parallel supporting lines of $C$ through $u$ and $v$.

Then $C \subset P=M \cap N$, and the $P$ lengths of the sides of $D$ are at least one. We observe also that $C$ is not

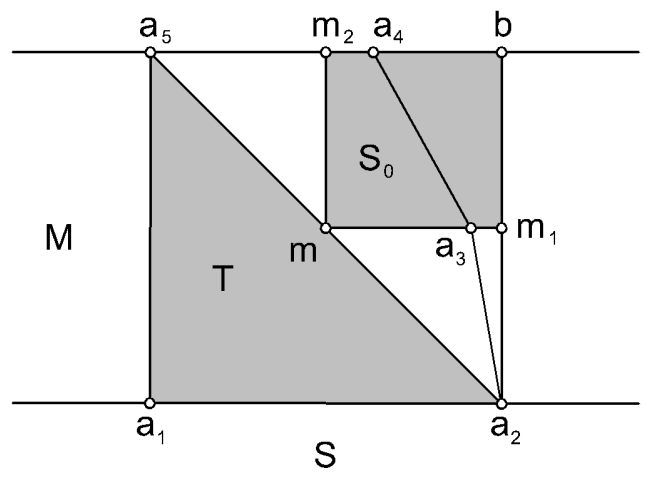

Figure 2 strictly convex.

\section{Proof of Theorems 1 and 2 when $Q=\operatorname{conv}\left\{a_{1}, a_{2}, \ldots, a_{7}\right\}$ is a hexagon}

Let us assume that $Q=\left[a_{1}, a_{2}, \ldots, a_{6}\right]$ and $a_{7} \in \operatorname{int} Q$. Let $a_{i}=q_{i}$ for $i=$ $1,2, \ldots, 6, q_{7}=q_{1}$ and $q_{0}=q_{6}$.

We use the following terms and notations. For any $i, j, k, l$, where $1 \leq i, j, k, l \leq 6$ and $\{i, j\} \neq\{k, l\}, \alpha_{i}$ denotes the angle of $Q$ at $q_{i}, q_{i j}$ denotes the midpoint of the segment $\left[q_{i}, q_{j}\right]$, and $L_{i j, k l}$ denotes the straight line containing $q_{i j}$ and $q_{k l}$. We note that $q_{i}=q_{i i}$ and set $L_{i, k l}=L_{i i, k l}$ and $L_{i, k}=L_{i i, k k}$. In addition, $S_{i}=\left[q_{i}, q_{i+1}\right]$ for 
$i=1,2, \ldots, 6$ and $M_{i}$ denotes the maximal chord of $Q$ parallel to $S_{i}$ with minimal Euclidean distance from $S_{i}$.

If $\alpha_{i-1}+\alpha_{i}+\alpha_{i+1}$ is greater than $2 \pi$, equal to $2 \pi$ or less than $2 \pi$, where $i=$ $1,2, \ldots, 6$, we say that $q_{i}$ is a large, normal or small vertex of $Q$, respectively. Observe that $q_{i}$ and $q_{i+3}$ are either both normal, or one of them is large and the other one is small.

Note that $\alpha_{i}+\alpha_{i+1} \leq \pi$ implies that $Q$ is contained in a parallelogram with $S_{i}$ as side. From this it readily follows that there is a triangle $T_{i}$ inscribed in $Q$ with the following property: $S_{i}$ is a side of $T_{i}$ and $T_{i}$ has maximum area of all triangles inscribed in $Q$. In this case the theorems follow from Lemma 3, and so we assume that the sum of every two consecutive angles of $Q$ is greater than $\pi$.

Next, it is a simple matter to check that:

Case 1, every second vertex of $Q$ is large, or

Case 2, $Q$ has three consecutive vertices such that the second one is large and the two other ones are not small, or

Case 3, $Q$ has three consecutive vertices such that the second one is normal and the two other ones are not small.

Case 1. Let the large vertices be $q_{1}, q_{3}$ and $q_{5}$, and $b_{i}=q_{1}+q_{3}+q_{5}-2 q_{i}$ for $i=1,3,5$; cf. Figure 3. Then $Q \subset\left[b_{1}, b_{3}, b_{5}\right]$ and every maximal chord of $Q$ passes through $q_{1}, q_{3}$ or $q_{5}$. Let $Q_{i}$ denote the homothetic copy of int $Q$ with ratio $1 / 2$ and with $q_{i}$ as centre. Let $P_{i}=\left[q_{i}, q_{(i-1) i}, q_{(i-1)(i+1)}, q_{(i+1) i}\right]$ for $i=2,4,6, T_{2}=\left[q_{13}, q_{14}, q_{36}\right]$, $T_{4}=\left[q_{35}, q_{36}, q_{25}\right], T_{6}=\left[q_{15}, q_{25}, q_{14}\right]$ and $T=\left(q_{14}, q_{25}, q_{36}\right)$.

We assume that $\left.d_{(} q_{i}, q_{i+1}\right) \geq 1$ for each $i$. Then we need only to show that for any $p \in \operatorname{int} Q$,

$(*)_{i} \quad d_{Q}\left(p, q_{i}\right)<1$

for some $i$. Let $p \in \operatorname{int} Q$. We consider the position of $p$ with respect to certain polygons. By symmetry, we assume that $p \in Q_{1} \cup P_{2} \cup T_{2} \cup T$.

We have:

(1) $(*)_{1}$ for $p \in Q_{1}$;

(2) $(*)_{2}$ for $p \in P_{2}$;

(3) $(*)_{2}$ or $(*)_{4}$ or $(*)_{6}$ for $p \in T_{2}$;

(4) $(*)_{2}$ or $(*)_{4}$ or $(*)_{6}$ for $p \in T$.

The statements in (1) and (2) are easy to show, and also (3) with the condition that $d_{Q}\left(q_{2}, q_{14}\right)<1$ and $d_{Q}\left(q_{2}, q_{36}\right)<1$. We show (3) for $d_{Q}\left(q_{2}, q_{14}\right) \geq 1$ and $d_{Q}\left(q_{2}, q_{36}\right) \geq 1$. When exactly one of $d_{Q}\left(q_{2}, q_{14}\right)$ and $d_{Q}\left(q_{2}, q_{36}\right)$ is at least one, the proof is similar.

Let $\left\{s_{1}\right\}=L_{35,25} \cap\left[q_{13}, q_{15}\right]$ and $\left\{s_{2}\right\}=L_{15,25} \cap\left[q_{13}, q_{35}\right]$. From $d_{Q}\left(q_{1}, q_{2}\right) \geq 1$ and $d_{Q}\left(q_{2}, q_{3}\right) \geq 1$, we have that $q_{2}$ is in the parallelogram with vertices $q_{13}$, $\left(q_{1}+b_{5}\right) / 2, b_{5}$ and $\left(q_{3}+b_{5}\right) / 2$. Thus the set of points in $\left[q_{13}, q_{35}, q_{15}\right]$, at $Q$-distance less than one from $q_{2}$, is $\left[q_{13}, s_{1}, q_{25}, s_{2}\right] \backslash\left(\left[s_{1}, q_{25}\right] \cup\left[q_{25}, s_{2}\right]\right)$. Similar statements are obtained for $q_{4}$ and $q_{6}$. Let $\left\{w_{1}\right\}=L_{35,36} \cap\left[q_{13}, q_{14}\right],\left\{w_{2}\right\}=L_{15,14} \cap\left[q_{13}, q_{36}\right]$ 


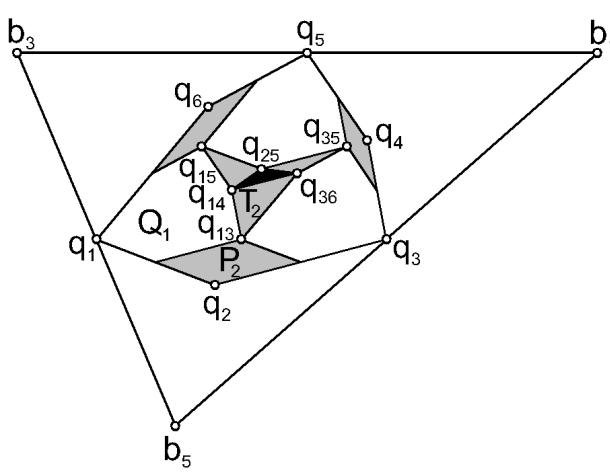

Figure 3

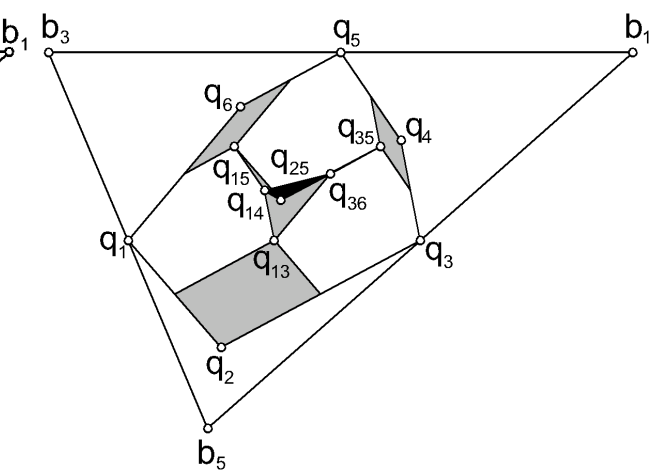

Figure 4

and $\{w\}=\left[q_{14}, w_{2}\right] \cap\left[q_{36}, w_{1}\right]$. As $d_{Q}\left(q_{2}, q_{14}\right) \geq 1$ and $d_{Q}\left(q_{2}, q_{36}\right) \geq 1$, it follows that $w_{1}, w_{2}$ and $w$ exist. Note that $p \in\left[q_{13}, w_{2}, w, w_{1}\right], p \in\left[q_{14}, w_{2}, q_{36}\right] \backslash\left[w_{2}, q_{14}\right]$ and $p \in\left[q_{14}, w_{1}, q_{36}\right] \backslash\left[w_{1}, q_{36}\right]$ imply $(*)_{2},(*)_{4}$ and $(*)_{6}$, respectively.

Next, we show (4). If $T \cap\left(T_{2} \cup T_{4} \cup T_{6}\right) \neq \emptyset$ then $T \subset T_{2} \cup T_{4} \cup T_{6}$, and our theorems follow from (3), and so we suppose that $T \cap\left(T_{2} \cup T_{4} \cup T_{6}\right)=\emptyset$; cf. Figures 3 and 4 . We need to distinguish between positions of lines that contain a vertex of $T$ and a side of some $T_{i}$. If $L_{15,25} \cap T=L_{35,25} \cap T=\emptyset$, we have $(*)_{2}$. If $L_{15,25} \cap T \neq \emptyset \neq L_{35,25} \cap T$ then $L_{13,14} \cap T=L_{15,14} \cap T=\emptyset$ and so we have $(*)_{4}$. Finally, we show that our hypotheses allow only these two possibilities.

Assume that $L_{15,25} \cap T \neq \emptyset$ and $L_{35,25} \cap T=\emptyset$. Let us take $v=\frac{1}{2}\left(\overrightarrow{q_{1} q_{6}}+\overrightarrow{q_{3} q_{2}}+\overrightarrow{q_{5} q_{4}}\right)$. As $\alpha_{i}+\alpha_{i+1}>\pi$ for $i=1,2, \ldots, 6, Q$ has a vertex $u$ such that the half line $V$ with endpoint $u$ and with tangential vector $v$ intersects int $Q$.

Assume that $u$ is a large vertex, say, $u=q_{1}$. Observe that $\overrightarrow{q_{53} q_{52}}=\frac{1}{2} \overrightarrow{q_{3} q_{2}}=$ $v-\frac{1}{2}\left(\overrightarrow{q_{1} q_{6}}+\overrightarrow{q_{5} q_{4}}\right)=v-\overrightarrow{q_{15} q_{46}}$. Let $n=q_{15}+v+\overrightarrow{q_{52} q_{36}}$. Then $\overrightarrow{q_{46} n}=\overrightarrow{q_{35} q_{36}}=\overrightarrow{q_{5} q_{56}}$, which implies $\overrightarrow{q_{45} n}=\overrightarrow{q_{5} q_{6}}$. For $i=1,2, \ldots, 6$, let $H_{i}$ denote the open half plane bounded by $L_{i 5,(i+1) 5}$ and containing $Q_{5}$. As $\overrightarrow{q_{45} n}=\overrightarrow{q_{5} q_{6}}, n \in H_{3} \cap H_{4} \cap H_{5}$. From $V \cap$ int $Q \neq \emptyset$, we have $q_{15}+v \in H_{6} \cap H_{1}$. From $L_{15,25} \cap T \neq \emptyset$, we obtain $q_{36} \in H_{6} \cap H_{1}$. This implies $n \in H_{6} \cap H_{1}$. Let $D=\operatorname{cl}\left(H_{3} \cap H_{4} \cap H_{5} \cap H_{6} \cap H_{1}\right)$. Then $n \in D$, and we have $d_{D}\left(q_{5}, q_{6}\right)<1$. As the maximal chords of $Q$ and $D$ in the direction of $\overrightarrow{q_{5} q_{6}}$ coincide, we have also $d_{Q}\left(q_{5}, q_{6}\right)<1$.

If $u$ is a small vertex, a similar consideration yields the contradiction.

Case 2. Let $q_{2}$ be large and $q_{1}$ and $q_{3}$ be not small. We show that $d_{C}\left(q_{i}, q_{j}\right) \leq 1$, and if $C$ is strictly convex then $d_{C}\left(q_{i}, q_{j}\right)<1$ for some $i \neq j$.

Recall that $S_{i}=\left[q_{i}, q_{i+1}\right]$ and $M_{i}$ is the maximal chord of $Q$ parallel to $S_{i}$ with the minimal Euclidean distance from $S_{i}$. Note that, as the sum of any two consecutive angles of $Q$ is greater than $\pi$, every maximal chord of $Q$ intersects $S_{j}$ and $S_{j+3}$ 
for some $j \in\{1,2,3\}$. If $M_{i}$ intersects $S_{j}$ and $S_{j+3}$, we say that $M_{i}$ is $a j$-type maximal chord. Observe that $M_{j}$ is not $j$-type and $M_{6}$ is not 3 -type. If $M_{6}$ and $M_{3}$ are 1-type and 2-type, respectively, then we observe that $q_{2}$ is not a small vertex; a contradiction. Hence, we have twelve possibilities depending on the types of $M_{1}$, $M_{2}, M_{3}$ and $M_{6}$. Let $\left\{d_{1}\right\}=L_{5,6} \cap L_{1,2},\left\{d_{2}\right\}=L_{6,1} \cap L_{2,3},\left\{d_{3}\right\}=L_{1,2} \cap L_{3,4}$ and $\left\{d_{4}\right\}=L_{2,3} \cap L_{4,5}$.

i) $M_{3}$ and $M_{6}$ are 1-type, $M_{1}$ is 2-type and $M_{2}$ is 3-type.

If $\left|q_{1} d_{2}\right|<\left|q_{1} q_{6}\right|$ then it follows from the type of $M_{1}$ that $d_{Q}\left(q_{1}, q_{2}\right)<1$. Similarly, $\left|q_{2} d_{3}\right|<\left|q_{1} q_{2}\right|$ implies $d_{Q}\left(q_{2}, q_{3}\right)<1$, and $\left|q_{3} d_{4}\right|<\left|q_{2} q_{3}\right|$ implies $d_{Q}\left(q_{3}, q_{4}\right)<1$. Assume that $\left|q_{1} d_{2}\right| \geq\left|q_{1} q_{6}\right|,\left|q_{2} d_{3}\right| \geq\left|q_{1} q_{2}\right|$ and $\left|q_{3} d_{4}\right| \geq\left|q_{2} q_{3}\right|$. Let $f_{1}$ be the intersection of $L_{1,2}$ and the line through $q_{6}$ parallel to $L_{3,4}, f_{2}$ be the intersection of $L_{2,3}$ and the line through $q_{1}$ parallel to $L_{3,4}$, and $g$ be the intersection of $L_{1,2}$ and the line through $q_{4}$ parallel to $L_{1,6}$; cf. Figure 5 . Since $q_{3}$ is not small and $\left|q_{3} d_{4}\right| \geq\left|q_{2} q_{3}\right|$, we have $\left|q_{3} q_{4}\right| \geq\left|q_{3} d_{3}\right|$. From $\left|q_{2} d_{3}\right| \geq\left|q_{1} q_{2}\right|$, we obtain that $\left|q_{3} d_{3}\right| \geq\left|q_{1} f_{2}\right|$. As $\left|q_{1} d_{2}\right| \geq\left|q_{1} q_{6}\right|$ and $Q$ is nondegenerate, we have also $\left|q_{1} f_{2}\right|>$ $\left|q_{6} f_{1}\right|$, and so $2\left|q_{6} f_{1}\right|<\left|q_{4} d_{3}\right|$. Since $2\left|q_{6} q_{1}\right|<\left|q_{4} g\right|$ and $M_{6}$ is 1-type, we obtain that $d_{Q}\left(q_{1}, q_{6}\right)<1$.

If $M_{3}$ and $M_{6}$ are 2-type, $M_{1}$ is 3-type and $M_{2}$ is 1-type then a similar argument yields $d_{Q}\left(q_{i-1}, q_{i}\right)<1$ for some $i \in\{1,2,3,4\}$.

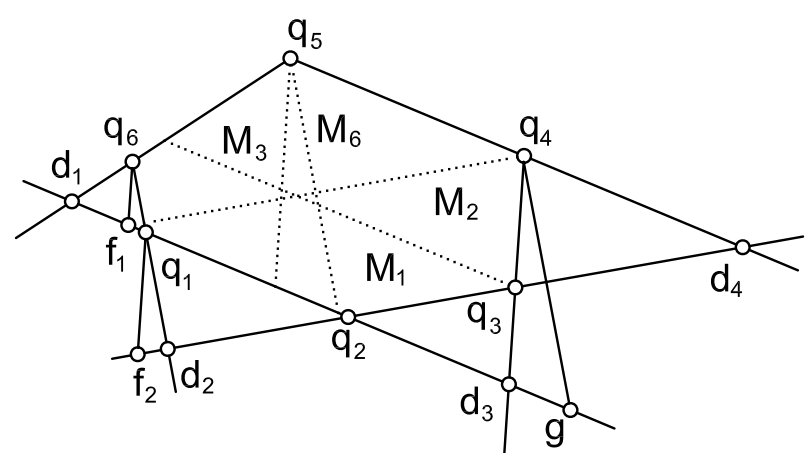

Figure 5

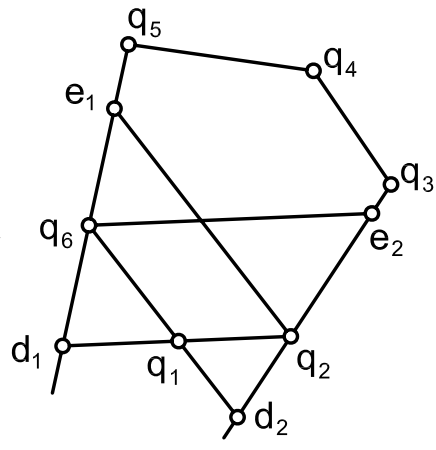

Figure 6

ii) $M_{6}$ and $M_{1}$ are 2-type.

Let $e_{1}$ denote the intersection of $S_{5}$ and the line through $q_{2}$ parallel to $S_{6}$, and $e_{2}$ denote the intersection of $S_{2}$ and the line through $q_{6}$ parallel to $S_{1}$; cf. Figure 6 . Since $M_{6}$ and $M_{1}$ are 2-type, $e_{1}$ and $e_{2}$ exist. Observe that $\left|d_{1} q_{1}\right| \leq\left|q_{1} q_{2}\right|$ or $\left|d_{2} q_{1}\right| \leq$ $\left|q_{1} q_{6}\right|$. If $\left|d_{1} q_{1}\right| \leq\left|q_{1} q_{2}\right|$, we have $2\left|q_{1} q_{6}\right| \leq\left|q_{2} e_{1}\right|$ and thus $d_{C}\left(q_{1}, q_{6}\right) \leq d_{Q}\left(q_{1}, q_{6}\right) \leq$ 1. Similarly, from $\left|d_{2} q_{1}\right| \leq\left|q_{1} q_{6}\right|$, we have $d_{C}\left(q_{1}, q_{2}\right) \leq 1$. A detailed analysis shows that, under the assumption that $C$ is strictly convex and $d_{C}\left(q_{i}, q_{j}\right) \geq 1$ for every $i \neq j, Q$ is an affine regular hexagon. Note that in this case all the vertices of $Q$ 
are normal $\left(\alpha_{i-1}+\alpha_{i}+\alpha_{i+1}=2 \pi\right.$ for $\left.i=1,2, \ldots, 6\right)$, but we assumed that $Q$ has a small vertex, a contradiction.

If $M_{1}$ and $M_{2}$ are 3-type, or $M_{2}$ and $M_{3}$ are 1-type then a similar argument shows our theorems. Hence, we have examined all the possibilities for the types of $M_{1}$, $M_{2}, M_{3}$ and $M_{6}$.

Case 3. Let $q_{2}$ be normal and $q_{1}, q_{3}$ be not small. As $\alpha_{1}+\alpha_{2}+\alpha_{3}=2 \pi, L_{6,1}$ and $L_{3,4}$ are parallel. Since relative distance is affine invariant, we assume that the triangle $\left[q_{1}, q_{3}, q_{5}\right]$ is regular and also that $\angle q_{6} q_{1} q_{3} \leq 90^{\circ}$. Let $b_{i}=q_{1}+q_{3}+q_{5}-2 q_{i}$ for $i=1,3,5$. Since $Q$ is convex and $q_{1}$ and $q_{5}$ are not small, $\left\{q_{2}, q_{4}, q_{6}\right\} \subset\left[b_{1}, b_{3}, b_{5}\right]$. Let $f=\left(q_{3}+b_{1}\right) / 2$ and $L$ be the line containing $q_{13}$ and parallel to $L_{1,6}$.

Subcase 3.1, $q_{2} \notin L$. Let $d=$ $\left(q_{3}+b_{5}\right) / 2$. We show that if $d_{Q}\left(q_{i}, q_{i+1}\right) \geq 1$ for every $i$ then $q_{2} \in\left[q_{1}, b_{5}, d\right] \backslash\left[q_{1}, d\right], q_{4} \in$ $\left[q_{5}, f, b_{1}\right]$ and $q_{6} \in\left[q_{15}, q_{5}, b_{3}\right]$. This yields $\frac{\pi}{6}<\angle q_{3} q_{1} q_{2}, \frac{\pi}{6} \leq$ $\angle q_{3} q_{5} q_{4}$ and that $q_{3}$ is a large vertex, a contradiction. In the case $\left|q_{1} q_{6}\right| \leq\left|q_{3} q_{4}\right|$, our arguments are deduced from Figure 7 with $k=q_{13}+\frac{1}{2} \overrightarrow{q_{6} q_{1}}$; $r=q_{1}+\frac{1}{2} \overrightarrow{q_{5} q_{13}} ; s=r+\frac{1}{2} \overrightarrow{q_{13} k} ;$ $c=\left(q_{1}+q_{5}+b_{3}\right) / 3$ and $t$ being the intersection of the line containing $\left[q_{15}, s\right]$ and the line containing $\left[q_{1}, c\right]$. We argue simi-

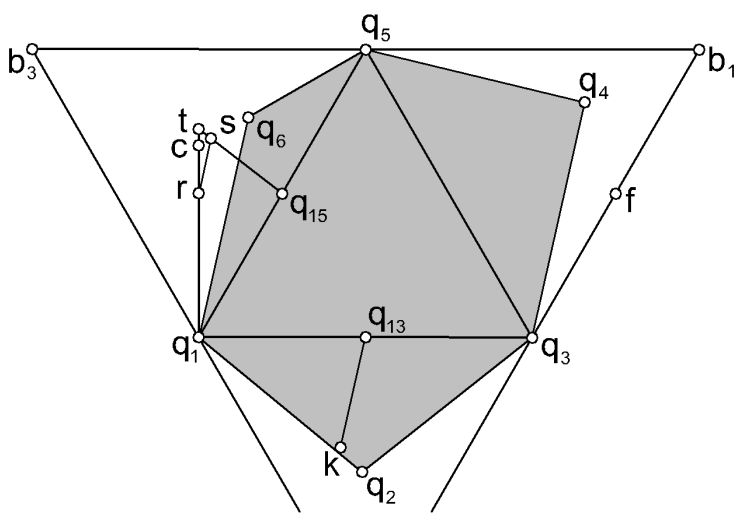

Figure 7 larly if $\left|q_{1} q_{6}\right| \geq\left|q_{3} q_{4}\right|$.

Subcase 3.2, $q_{2} \in L$. Observe that $q_{3} \in M_{1}$ and $M_{1} \cap\left(\left(q_{1}, q_{6}\right) \cup S_{5}\right) \neq \emptyset$. If $M_{1} \cap$ $\left(q_{1}, q_{6}\right) \neq \emptyset$ then $d_{C}\left(q_{1}, q_{2}\right) \leq d_{Q}\left(q_{1}, q_{2}\right)=1$. Moreover, if $d_{C}\left(q_{1}, q_{2}\right)=1$ then $M_{1}$ is maximal also in $C$, which implies that $C$ is not strictly convex. Similarly, if $M_{2} \cap\left(q_{3}, q_{4}\right) \neq \emptyset$ then $d_{C}\left(q_{2}, q_{3}\right) \leq 1$, and $d_{C}\left(q_{2}, q_{3}\right)<1$ or $C$ is not strictly convex.

Assume that $M_{1} \cap S_{5} \neq \emptyset \neq M_{2} \cap S_{3}$. Let $w$ be the intersection of $L_{1,6}$ and the line containing $M_{1}$. Observe that $\left[q_{1}, q_{3}, w\right]$ is a homothetic copy of $\left[q_{1}, q_{2}, q_{13}\right]$ of ratio -2 , and $2\left|q_{13} q_{2}\right| \geq\left|q_{1} q_{6}\right|$. Similarly, we obtain that $2\left|q_{13} q_{2}\right| \geq\left|q_{3} q_{4}\right|$. As above, this and $d_{Q}\left(q_{1}, q_{6}\right) \geq 1$ imply that $q_{6} \in\left[q_{15}, q_{5}, b_{3}\right], q_{4} \in\left[q_{5}, f, b_{1}\right]$ and $\frac{\pi}{6} \leq \angle q_{2} q_{1} q_{3}$. Since $q_{1}$ is not a small vertex, it follows that $\angle q_{2} q_{1} q_{3}=\frac{\pi}{6}, q_{4} \in\left[q_{5}, f\right]$, $q_{6} \in\left[b_{3}, q_{15}\right]$ and $M_{1}=\left[q_{3}, q_{6}\right]$. Let $\{x\}=L_{1,3} \cap L_{4,5}$. Notice that $\left[q_{3}, q_{4}, x\right]$ is a homothetic copy of $\left[q_{1}, q_{2}, q_{13}\right]$ of ratio $-2,\left|q_{3} q_{4}\right|=2\left|q_{2} q_{13}\right|$ and $\left|q_{1} q_{6}\right|=2\left|q_{2} q_{13}\right|$. Observe that $q_{1} \in M_{5}, d_{Q}\left(q_{5}, q_{6}\right)=1$ and $M_{5} \cap S_{4} \neq \emptyset$. Let $\{y\}=M_{5} \cap S_{4}$. As $d_{C}\left(q_{5}, q_{6}\right) \leq d_{Q}\left(q_{5}, q_{6}\right)$, we may assume that $d_{C}\left(q_{5}, q_{6}\right)=1$. In this case, $\left[q_{1}, y\right]$ is a maximal chord of $C$. If $y \neq q_{4}$ then $y \in\left(q_{4}, q_{5}\right)$ and $C$ is not strictly convex. 
If $y=q_{4}, Q$ is a regular hexagon. Let $c$ be the centre of $Q$. If $p \neq c$ is a point of $\left[q_{i}, q_{i+1}, c\right]$ then $d_{Q}\left(q_{i}, p\right)<1$ or $d_{Q}\left(q_{i+1}, p\right)<1$. Hence, the only point of $Q$ at $Q$-distance at least one from every vertex of $Q$ is the centre of $Q$.

The last case is $a_{7} \in$ bd $Q$. We regard $Q$ as a degenerate heptagon and prove Theorems 1 and 2 in Section 3.

\section{Proof of Theorems 1 and 2 when $Q=\operatorname{conv}\left\{a_{1}, a_{2}, \ldots, a_{7}\right\}$ is not a hexagon}

We assume that no triangle, of the largest possible area inscribed in $Q$, has a side that coincides with a side of $Q$; otherwise, Theorems 1 and 2 follow from Lemma 5.

Case $1, Q=\left[a_{1}, a_{2}, \ldots, a_{7}\right]$.

Let $T$ be a triangle of the largest possible area inscribed in $Q$ such that the vertices of $T$ are also vertices of $Q$. Assume that $T=\left[a_{1}, a_{3}, a_{6}\right]$. Since relative distance is affine invariant, we assume that $T$ is a regular triangle. Let $b_{i}=a_{1}+a_{3}+a_{6}-2 a_{i}$ for $i=1,3,6$. As $T$ is a triangle of the largest area and $Q$ is convex, we have $a_{2} \in\left[a_{1}, b_{6}, a_{3}\right],\left\{a_{4}, a_{5}\right\} \subset\left[a_{3}, b_{1}, a_{6}\right]$ and $a_{7} \in\left[a_{6}, b_{3}, a_{1}\right]$.

Let $s_{1}=\left(a_{3}+a_{6}\right) / 2, s_{2}=\left(a_{3}+b_{1}\right) / 2, s_{3}=$ $\left(b_{1}+a_{6}\right) / 2, t_{1}=\left(a_{6}+a_{1}\right) / 2, t_{2}=\left(a_{6}+b_{3}\right) / 2$ and $t_{3}=\left(b_{3}+a_{1}\right) / 2$. If $d_{Q}\left(a_{3}, a_{4}\right)<1$ or $d_{Q}\left(a_{5}, a_{6}\right)<1$, we are done, and so, we have that $\left\{a_{4}, a_{5}\right\} \subset\left[s_{1}, s_{2}, b_{1}, s_{3}\right]$. Note that the convexity of $Q$ implies $d_{Q}\left(a_{4}, a_{5}\right) \leq 1$ and thus, Theorem 1; cf. Figure 8. To prove Theorem 2, we assume that $d_{Q}\left(a_{i}, a_{i+1}\right) \geq d_{C}\left(a_{i}, a_{i+1}\right) \geq 1$ for every $i$. Then $d_{C}\left(a_{4}, a_{5}\right)=1$ and $a_{4}, a_{5}$ are on parallel sides of the rhombus $\left[s_{1}, s_{2}, b_{1}, s_{3}\right]$. We assume that $a_{4} \in\left[s_{1}, s_{2}\right]$ and $a_{5} \in\left[b_{1}, s_{3}\right]$. Let $L_{1}$ be the line through $a_{1}$ and $a_{3}$, and $L_{2}$ be the line through $b_{1}$ and $b_{3}$. Let $H_{1}$ and $H_{2}$ be the open half planes containing $\left(a_{2}, a_{6}\right)$ and bounded by the lines $L_{1}$ and $L_{2}$, respectively.

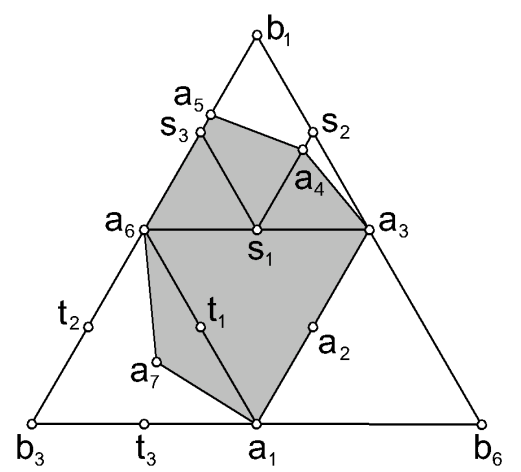

Figure 8 Observe that there are points $u \in\left(a_{5}, a_{6}\right)$ and $v \in\left(a_{1}, a_{3}\right)$ such that $\overrightarrow{u v} \| \overrightarrow{a_{3}, a_{4}}$. As $d_{C}\left(a_{3}, a_{4}\right) \geq 1,[u, v]$ is a maximal chord of $C$, and so, $C \subset H_{1} \cap H_{2}$.

Since $C \subset H_{1}, a_{2} \in\left[a_{1}, a_{3}\right]$. Thus $a_{2}=\left(a_{1}+a_{3}\right) / 2$ and $d_{C}\left(a_{1}, a_{2}\right)=d_{C}\left(a_{2}, a_{3}\right)=1$. Due to $d_{C}\left(a_{1}, a_{3}\right)=2$, there are parallel supporting lines $L_{3}$ and $L_{4}$ of $C$ through $a_{1}$ and $a_{3}$. Let $a_{1} \in L_{3}$ and $a_{3} \in L_{4}$. Let $P$ be the parallelogram bounded by $L_{1}$, $L_{2}, L_{3}, L_{4}$. Clearly, $C \subset P$.

We show that the $P$-length of every side of $Q$ is at least one. We verify that $d_{P}\left(a_{7}, a_{1}\right) \geq 1$ and $d_{P}\left(a_{6}, a_{7}\right) \geq 1$, as the other inequalities are trivial. 
From $d_{Q}\left(a_{6}, a_{7}\right) \geq 1$ and $d_{Q}\left(a_{7}, a_{1}\right) \geq 1$ we have $a_{7} \in\left[t_{1}, t_{2}, b_{3}, t_{3}\right]$. This implies $d_{P}\left(a_{7}, a_{1}\right) \geq 1$. Let $x$ be the vertex of $P$ on $\left[a_{6}, b_{3}\right]$. As $s_{3} \in Q \subset P$ and $\left[a_{1}, a_{3}\right]$ is a side of $P$, we have $x \in\left[a_{6}, t_{2}\right]$. Let $\{t\}=\left[a_{1}, t_{2}\right] \cap\left[t_{1}, t_{3}\right]$. Observe that $a_{7} \in\left[t_{1}, t, t_{2}\right]$. If $a_{7} \notin\left[t_{1}, t\right] \cup\left[t, t_{2}\right]$ then $d_{Q}\left(a_{6}, a_{7}\right)<1$; a contradiction. If $a_{7} \in\left[t_{1}, t\right] \cup\left[t, t_{2}\right]$ then $d_{P}\left(a_{6}, a_{7}\right)=1$.

Case 2, $Q$ is a triangle or $Q$ contains a quadrangle $R$ such that, with a suitable labelling of the $a_{i}$ 's, $R=\left[a_{1}, a_{2}, a_{3}, a_{4}\right]$ and card (int $\left.R \cap\left\{a_{5}, a_{6}, a_{7}\right\}\right) \geq 2$.

The proof in Case 2 is a refined version of the proof of Lemma 3 in [2], hence we omit it.

\section{Applications}

Bateman and Erdös [1] asked what is the smallest diameter of a set of $k$ points in $\mathbb{E}^{n}$ with pairwise distances at least one. In their paper they showed that, for seven points in the plane, the smallest diameter is two. The extension of this result for normed planes was, to our knowledge, first proposed by K. Bezdek (Problem Session, Workshop on Discrete Geometry and Convexity, Auburn University, Auburn, Alabama, USA, April, 2000). In 2005, Brass and Swanepoel conjectured that, in every normed plane, if $S$ is a set of seven points with pairwise distances at least one, then the diameter of $S$ is at least two (cf. Problem 10 on p. 71, [3]). In this section, we consider the question of Bateman and Erdös, and extend their result to normed planes. We prove also the conjecture of Brass and Swanepoel.

Let $C \in \mathcal{M}$ and $k \geq 2$. Compactness arguments show the existence of the smallest diameter $g_{k}(C)$ of a set of $k$ points in the normed plane for a unit disk $C$ such that the pairwise distances of the points are at least one. Let $g_{k}=\min _{C \in \mathcal{M}}\left\{g_{k}(C)\right\}$ and $G_{k}=\max _{C \in \mathcal{M}}\left\{g_{k}(C)\right\}$. By a Blaschke type theorem these values exist.

To formulate our next theorem, we introduce for any $C \in \mathcal{M}$ and $k \geq 2, \bar{f}_{k}(C)=$ $\max \left\{f_{k}(D) \mid D \in \mathcal{C}\right.$ and $\left.\frac{1}{2}(D-D)=C\right\}$. In other words, we consider the maximum over the ovals 'generating' the normed plane with unit disk $C$.

Theorem 6 Let $k \geq 2$ and $C \in \mathcal{M}$. Then $g_{k}(C) \cdot \bar{f}_{k}(C)=2$.

Proof. We show only that $\bar{f}_{k}(C) \geq 2 / g_{k}(C)$, since the opposite direction is simple. Let $a_{1}, a_{2}, \ldots, a_{k}$ be points in the normed plane with unit disk $C$ at pairwise distances at least one such that $\operatorname{diam}\left(\left\{a_{1}, a_{2}, \ldots, a_{k}\right\}\right)=g_{k}(C)$. Let $b_{i}=\frac{2}{g_{k}(C)} a_{i}$ for $i=1,2, \ldots, k$. The pairwise distances of the points $b_{i}$ are at least $2 / g_{k}(C)$ and $\operatorname{diam}\left(\left\{b_{1}, b_{2}, \ldots, b_{k}\right\}\right)=2$. According to [8], there is a plane convex body $D$ of constant width two containing $b_{i}$ for $i=1,2, \ldots, k$. As $D$ is of constant width two, we have $\frac{1}{2}(D-D)=C$. Hence, $D$ contains $k$ points at pairwise $D$-distances at least $2 / g_{k}(C)$. 
Using Theorem 6 , we determine $g_{k}$ and $G_{k}$ for small values of $k$.

Theorem 7 Let $C \in \mathcal{M}$ and $g_{k}(C)$ be defined as above, $g_{k}=\min _{C \in \mathcal{M}}\left\{g_{k}(C)\right\}$ and $G_{k}=\max _{C \in \mathcal{M}}\left\{g_{k}(C)\right\}$.

7.1 If $k \leq 4$ then $g_{k}=1$.

$7.2 \quad g_{5}=\frac{\sqrt{5}+1}{2}$.

$7.3 g_{6}=\frac{5+\sqrt{5}}{4}$, and $g_{6}(C)=\frac{5+\sqrt{5}}{4}$ if, and only if, $C$ is an affine regular ten-gon.

$7.4 g_{7}=g_{8}=g_{9}=2$, and $g_{9}(C)=2$ if, and only if, $C$ is a parallelogram.

$7.5 G_{2}=G_{3}=1$.

$7.6 G_{4}=\sqrt{2}$.

$7.7 G_{5}=G_{6}=G_{7}=2$ and $g_{7}(C)=2$.

Proof. The statements in 7.1 to 7.6 follow from Theorem 6 and from results in [1], [2], [5], [7] and [10]. We prove only 7.7.

First, we show that $\bar{f}_{5}(P)=1$ for any parallelogram $P$. Observe that $f_{5}(P)=1$. Hence, it is enough to show that if $C$ is an oval of constant width two in the normed plane with unit disk $P$ then $C$ is a translate of $P$. Let $P=\left[a_{1}, a_{2}, a_{3}, a_{4}\right]$. Let $L_{1}$ and $L_{2}$ be the supporting lines of $C$ parallel to $\overrightarrow{a_{1} a_{2}}$ such that the translate of $L_{1}$ by $\overrightarrow{a_{1} a_{4}}$ is $L_{2}$. Let $\left[b_{1}, b_{2}\right]=C \cap L_{1}$ and $\left[b_{3}, b_{4}\right]=C \cap L_{2}$ such that $\overrightarrow{b_{1} b_{2}}$ and $\overrightarrow{b_{4} b_{3}}$ are positive multiples of $\overrightarrow{a_{1} a_{2}}$. Let $c_{3}=b_{1}+\overrightarrow{a_{1} a_{3}}$ and $c_{4}=b_{2}+\overrightarrow{a_{2} a_{4}}$. Since $C$ is of constant width two, we have $\left[c_{3}, c_{4}\right] \subset\left[b_{3}, b_{4}\right]$. Observe that $\left|c_{3} c_{4}\right|=2\left|a_{1} a_{2}\right|-\left|b_{1} b_{2}\right|$. As $\left|b_{3} b_{4}\right| \leq\left|a_{1} a_{2}\right|$, this implies that $\left|b_{1} b_{2}\right|=\left|b_{3} b_{4}\right|=\left|a_{1} a_{2}\right|, c_{3}=b_{3}$ and $c_{4}=b_{4}$. Hence, $D=\left[b_{1}, b_{2}, b_{3}, b_{4}\right]$ is a translate of $P$. As $D \subset C$ and $C$ is an oval of constant width two, we have $C=D$.

Due to [11], every oval contains five points at pairwise relative distances at least one. Hence, $g_{5}(C)=2 / \bar{f}_{5}(C) \leq 2$ for any $C \in \mathcal{M}$. We have shown that $g_{5}(P)=2$ for any parallelogram $P$. Consequently, we have $G_{5}=2$. In [5], the authors show that every centrally symmetric oval contains seven points at pairwise relative distances at least one. Thus $\bar{f}_{7}(C) \geq 1$ for any $C \in \mathcal{M}$. This implies that $G_{7} \leq 2$. Now $G_{5} \leq G_{6} \leq G_{7}$ yields that $G_{5}=G_{6}=G_{7}=2$. Since $G_{7}=g_{7}=2$, we obtain that $g_{7}(C)=2$ for every $C \in \mathcal{M}$.

The next theorem is based on Theorem 2. As its proof is similar to that of Theorem 7 , we omit it.

Theorem 8 Let $a_{1}, a_{2}, \ldots, a_{7}$ be points, at pairwise distances at least one and with diameter two, in the normed plane with unit disk $C$. Let $Q=\operatorname{conv}\left\{a_{1}, a_{2}, \ldots, a_{7}\right\}$. 8.1 If $C$ is strictly convex then $a_{1}, a_{2}, \ldots, a_{7}$ are the vertices and the centre of an affine regular hexagon.

8.2 If card $\left(\operatorname{bd} Q \cap\left\{a_{1}, a_{2}, \ldots, a_{7}\right\}\right) \neq 6$ then there is a parallelogram $P$ such that 
$C \subset P$ and the pairwise distances of $a_{1}, a_{2}, \ldots, a_{7}$ are at least one in the normed plane with unit disk $P$.

Acknowledgements. The authors are indebted to K. Bezdek for raising a question that led to Theorems 6, 7 and 8, and to T. Bisztriczky for his helpful remarks.

\section{References}

[1] P. Bateman and P. Erdős, Geometrical extrema suggested by a lemma of Besicovitch, Amer. Math. Monthly 58 (1951) 306-314.

[2] K. Böröczky and Z. Lángi, On the relative distances of six points in a plane convex body, Stud. Sci. Math. Hungar. 42 (2005) 253-264.

[3] P. Brass, W. Moser and J. Pach, Research problems in discrete geometry, Springer, New York, 2005.

[4] K. Doliwka and M. Lassak, On relatively short and long sides of convex pentagons, Geom. Dedicata 56 (1995) 221-224.

[5] P. G. Doyle, J. C. Lagarias and D. Randall, Self-packing of centrally symmetric convex bodies in $R^{2}$, Discrete Comput. Geom. 8 (1992) 171-189.

[6] S. Gołąb, Some metric problems of the geometry of Minkowski, Trav. Acad. Mines Cracovie 6 (1932) 1-79.

[7] H. Groemer, Abschätzungen für die Anzahl der Konvexen Körper, die eine konvexen Körper berühren, Monatsh. Math. 65 (1961) 74-81.

[8] P. J. Kelly, On Minkowski bodies of constant width, Bull. Amer. Math. Soc. 55 (1949) 1147-1150.

[9] Z. Lángi, On the relative lengths of sides of convex polygons, Stud. Sci. Math. Hungar. 40 (2003) 115-120.

[10] Z. Lángi and M. Lassak, Relative distance and packing a body by homothetical copies, Geombinatorics XIII (2003) 29-40.

[11] M. Lassak, On five points in a plane convex body pairwise in at least unit relative distances, Coll. Math. Soc. János Bolyai 63 (1991) 245-247.

Antal Joós

Dept. of Geom.

Eötvös University

Budapest, Hungary

e-mail:ajoos@kac.poliod.hu
Zsolt Lángi

Dept. of Math. and Stats. University of Calgary

Calgary, Canada

e-mail: zlangi@math.ucalgary.ca 